\title{
Editorial
}

\section{Water and Health, Ecosystems and Drought}

Many of the articles in this issue have a water and health theme, and this is not unusual for our journal. Several articles cover emerging infectious diseases in the aquatic medium, the health of aquatic animals, and the influence of weather patterns and climate change on them. Taken as whole, the issue demonstrates the contributions that a focus on water, particularly wetlands and catchments, can make. By explicitly reintegrating ecosystems, social systems, and governance, water can be seen as both the context and the determinant for health promotion's healthy settings agenda (Parkes and Horwitz, 2009).

Two Australian articles in this issue exemplify the agenda. Sadgrove (2010) proposes a hypothesis concerning indigenous aquatic hunting practices and their control of cyanobacterial blooms. By bringing together ethnobotany, toxicology, and an understanding of Australian hydrology and water chemistry, the author acknowledges indigenous culture and offers a novel water treatment option.

Public attitudes in Australia to water recycling are examined by Mikhailovich (2010), whose commentary includes an argument for the adoption of the Convention on Biological Diversity's ecosystems approach, for the purposes of policy determination and public education. Our cover also shows the same theme with spiritual indigenous representations of iconic aquatic Australian animals.

This focus applies well on the Australian continent, a nation with its share of water issues and where, as Mikhailovich points out, a range of discourses has developed. These discourses account for different definitions of the water problem and therefore different versions of the solution. For example, adoption of a healthy settings discourse with the attendant principles of health promotion should ensure that human health and well-being remain central in water resource management. Prevention, and awareness of the systemic consequences of water resource development means that attention should be oriented towards hydrological regimes, and biota adapted to them, to guide decision-making.

Most Australian ecosystems are dominated by extraordinary climatic variability, and a boom and bust cycle that reflects the way that the continent receives, stores, and makes available water. Patterns of diversity and productivity follow very large, slow, infrequent, and unpredictable pulses of water. However, few of the discourses about water start with this premise. Instead, water resource development in Australia has been engineered in such a way as to provide fixed yields interannually.

When water resource management seeks to impose a stable demand upon a fluctuating supply, it does so by regulating the supply, not by adjusting the demand. But for the majority of Australian rivers and aquifers, a regulated supply cannot meet an unregulated demand. The same probably goes for an economy dependant upon it.

The health consequences of these economic imperatives might be both direct and indirect. Reservoirs and their irrigation channels become aseasonal sites for vector breeding and increased exposures to arboviral diseases. They also become routes for the transfer of sediment, nutrients, and pesticides into lower lying water bodies where people congregate. Increased groundwater abstraction exposes organic rich sulfidic soils, resulting in heavy metal plumes in groundwater, acidic surface waters, and the possibilities of exposures to smoke from peat fires. Fixed infrastructure, entrenched behaviors and unmet demand in the agriculture sector results in institutional failures and reduced individual capacities to cope with periods of water shortage- this runs contrary to the knowledge that droughts will occur in Australia. Psychosocial impacts and mental health issues associated with drought are of increasing concern (Sartore et al., 2008). Agricultural de- 
mands for water in the face of economic imperatives produce unsustainable broadscale land-use patterns, dictate the food we eat and the contaminants we consume with it, and contribute to sedentary lifestyles, together with the rise in noncommunicable diseases.

Many of these health outcomes are conjectural, and the degree to which interventions are applicable is contingent upon local conditions. Nevertheless, their possibilities demonstrate two important implications for governance in Australia. First, the expansive and cross-cutting nature of water resource management belies the sectoralized approach adopted for it, and challenges us to more appropriately account for water use, and to regulate demand to fit with the variabilities of the systems that make water available. Second, the health sector cannot be silent partners in this pursuit, where a closer attention to ecosystem services like that outlined in the Millennium Ecosystem Assessment is warranted.

Pierre Horwitz

Consortium for Health and Ecology, Edith Cowan University, School of Natural Sciences, Joondalup, Western Australia 6027, Australia

e-mail: horwitz@ecohealth.net; p.horwitz@ecu.edu.au

\section{REFERENCES}

Mikhailovich K (2010) New water, new meaning: engaging communities about water recycling. EcoHealth 6

Parkes M, Horwitz P (2009) Water, ecology and health: ecosystems as 'settings' for health and sustainability. Health Promotion International 24:94-102

Sadgrove N (2010) The influence of indigenous food procurement techniques on cyanobacteria populations in pre-European Australia: a potential small-scale water management tool. EcoHealth 6

Sartore G, Kelly B, Stain H, Albrecht G, Higginbotham N (2008) Control, uncertainty, and expectations for the future: a qualitative study of the impact of drought on a rural Australian community. Rural and Remote Health 8:950

Published online: March 9, 2010 